\title{
INEQUALITIES FOR SUMS OF RANDOM VARIABLES IN NONCOMMUTATIVE PROBABILITY SPACES
}

\author{
GHADIR SADEGHI AND MOHAMMAD SAL MOSLEHIAN
}

\begin{abstract}
In this paper, we establish an extension of a noncommutative Bennett inequality with a parameter $1 \leq r \leq 2$ and use it together with some noncommutative techniques to establish a Rosenthal inequality. We also present a noncommutative Hoeffding inequality as follows: Let $(\mathfrak{M}, \tau)$ be a noncommutative probability space, $\mathfrak{N}$ be a von Neumann subalgebra of $\mathfrak{M}$ with the corresponding conditional expectation $\mathcal{E}_{\mathfrak{N}}$ and let subalgebras $\mathfrak{N} \subseteq \mathfrak{A}_{j} \subseteq$ $\mathfrak{M}(j=1, \cdots, n)$ be successively independent over $\mathfrak{N}$. Let $x_{j} \in \mathfrak{A}_{j}$ be self-adjoint such that $a_{j} \leq x_{j} \leq b_{j}$ for some real numbers $a_{j}<b_{j}$ and $\mathcal{E}_{\mathfrak{N}}\left(x_{j}\right)=\mu$ for some $\mu \geq 0$ and all $1 \leq j \leq n$. Then for any $t>o$ it holds that
\end{abstract}

$$
\operatorname{Prob}\left(\left|\sum_{j=1}^{n} x_{j}-n \mu\right| \geq t\right) \leq 2 \exp \left\{\frac{-2 t^{2}}{\sum_{j=1}^{n}\left(b_{j}-a_{j}\right)^{2}}\right\} \text {. }
$$

1. Introduction. By a noncommutative probability space $(\mathfrak{M}, \tau)$ we mean a von Neumann algebra $\mathfrak{M}$ on a Hilbert space $\mathfrak{H}$ with unit element 1 equipped with a faithful normal finite trace $\tau$ such that $\tau(1)=1$. The modules $|x|$ of $x \in \mathfrak{M}$ are defined by continuous functional calculus as $|x|=\left(x^{*} x\right)^{1 / 2}$.

For each self-adjoint operator $x \in \mathfrak{M}$, there exists a unique spectral measure $E$ as a $\sigma$-additive mapping with respect to the strong operator topology from the Borel $\sigma$-algebra $\mathcal{B}(\mathbb{R})$ of $\mathbb{R}$ into the set of all orthogonal projections such that, for every Borel function $f: \sigma(x) \rightarrow \mathbb{C}$, the operator $f(x)$ is defined by

$$
f(x)=\int f(\lambda) d E(\lambda),
$$

2010 AMS Mathematics subject classification. Primary 46L53, 47A30, Secondary 60B20, 60B11.

Keywords and phrases. Noncommutative probability space, von Neumann algebra, noncommutative Rosenthal inequality, noncommutative Bennett inequality, noncommutative Hoeffding inequality.

Received by the editors on April 23, 2014. 
in particular,

$$
x=\int \lambda d E(\lambda)
$$

and

$$
\chi_{B}(x)=\int_{B} d E(\lambda)=E(B) .
$$

In addition,

$$
\tau\left(\chi_{[t, \infty)}(|x|)\right)=\tau\left(\chi_{[t, \infty)}(x)\right)+\tau\left(\chi_{[t, \infty)}(-x)\right) .
$$

Further, if $x \geq 0$ and $t>0$, then $\chi_{[t, \infty)}(x) t \leq x$. Hence, we get the inequality

$$
\tau\left(\chi_{[t, \infty)}(x)\right) \leq t^{-1} \tau(x),
$$

which is called the Markov inequality in the literature. For a self-adjoint element $x \in \mathfrak{M}$, it follows from the Markov inequality that

$$
\tau\left(\chi_{[t, \infty)}(x)\right)=\tau\left(\chi_{\left[e^{t}, \infty\right)}\left(e^{x}\right)\right) \leq e^{-t} \tau\left(e^{x}\right),
$$

from where we reach the exponential Chebyshev inequality as follows:

$$
\tau\left(\chi_{[t, \infty)}(x)\right) \leq e^{-t} \tau\left(e^{x}\right) .
$$

As in the commutative context, we use the notation $\operatorname{Prob}(x \geq t):=$ $\tau\left(\chi_{[t, \infty)}(x)\right)$.

For any Borel set $A \subseteq \mathbb{R}$, we define $\nu(A)=\tau(E(A))$. Then $\nu$ is a scalar-valued spectral measure for $x$ and $\nu(\mathbb{R})=1$. In addition,

$$
\tau(f(x))=\tau\left(\int f(\lambda) d E(\lambda)\right)=\int f(\lambda) d \tau(E(\lambda))=\int f(\lambda) d \nu(\lambda) .
$$

By the measurable functional calculus [4] there is a $*$-homomorphism $\pi: L^{\infty}(\nu) \rightarrow \mathfrak{M}$ depending on $x$ such that $\pi(f)=f(x)$ for all $f \in L^{\infty}(\nu)$.

For $1 \leq p<\infty, L^{p}(\mathfrak{M}, \tau)$ is defined as the completion of $\mathfrak{M}$ with respect to the norm $\|x\|_{p}=\tau\left(|x|^{p}\right)^{1 / p}$. Important special cases of these noncommutative spaces are the usual $L^{p}$-spaces and the Schatten $p$ classes. For further information, we refer the reader to $[\mathbf{1 1}, \mathbf{1 3}, \mathbf{1 6}, \mathbf{2 0}]$. Let $x \in \mathfrak{M}$ be positive. For $p \geq 1$ and positive $x \in \mathfrak{M}$, from (1.3), we 
have

$$
\begin{aligned}
\|x\|_{p}^{p}=\tau\left(x^{p}\right) & =\int_{0}^{\infty} \lambda^{p} d \nu(\lambda) \\
& =\int_{0}^{\infty} p t^{p-1} \nu([t, \infty)) d t \\
& =\int_{0}^{\infty} p t^{p-1} \tau\left(\chi_{[t, \infty)}(x)\right) d t .
\end{aligned}
$$

Let $\mathcal{P}$ be the lattice of projections of $\mathfrak{M}$. Set $p^{\perp}=1-p$ for $p \in \mathcal{P}$. Given a family of projections $\left(p_{\lambda}\right)_{\lambda \in \Lambda} \subseteq \mathcal{P}$, we denote by $\vee_{\lambda \in \Lambda} p_{\lambda}$ (respectively, $\wedge_{\lambda \in \Lambda} p_{\lambda}$ ) the projection from $\mathfrak{H}$ onto the closed subspace generated by $p_{\lambda}(\mathfrak{H})$ (respectively, onto the subspace $\left.\cap_{\lambda \in \Lambda} p(\mathfrak{H})\right)$. Consequently, $\left(\vee_{\lambda \in \Lambda} p_{\lambda}\right)^{\perp}=\wedge_{\lambda \in \Lambda} p_{\lambda}^{\perp}$. Two projections $p$ and $q$ are said to be equivalent if there exists a partial isometry $u \in \mathfrak{M}$ such that $u^{*} u=p$ and $u u^{*}=q$. In this case, we write $p \sim q$. If $p$ is equivalent to a projection $q_{1} \leq q$, we write $p \prec q$. $[12])$.

We need the following elementary properties of projections (see

Lemma 1.1. Let $p$ and $q$ be two projections of $\mathfrak{M}$. Then:

(i) $p \vee q-q \sim p-p \wedge q$.

(ii) If $p \wedge q=0$ then $p \prec q^{\perp}$.

(iii) If $p$ and $q$ are equivalent projections in $\mathfrak{M}$, then $\tau(p)=\tau(q)$.

(iv) If $\left(p_{\lambda}\right)_{\lambda \in \Lambda}$ is a family of projections in $\mathfrak{M}$, then $\tau\left(\vee_{\lambda \in \Lambda} p_{\lambda}\right) \leq$ $\sum_{\lambda \in \Lambda} \tau\left(p_{\lambda}\right)$.

Let $\mathfrak{N}$ be a von Neumann subalgebra of $\mathfrak{M}$. Then there exists a map $\mathcal{E}_{\mathfrak{N}}: \mathfrak{M} \rightarrow \mathfrak{N}$ satisfying the following properties:

(i) $\mathcal{E}_{\mathfrak{N}}$ is a normal contraction positive map projecting $\mathfrak{M}$ onto $\mathfrak{N}$;

(ii) $\mathcal{E}_{\mathfrak{N}}(a x b)=a \mathcal{E}_{\mathfrak{N}}(x) b$ for any $x \in \mathfrak{M}$ and $a, b \in \mathfrak{N}$;

(iii) $\tau \circ \mathcal{E}_{\mathfrak{N}}=\tau$.

Moreover, $\mathcal{E}_{\mathfrak{N}}$ is the unique map satisfying (i) and (ii). The map $\mathcal{E}_{\mathfrak{N}}$ is called the conditional expectation of $\mathfrak{M}$ with respect to $\mathfrak{N}$. We say that two subalgebras $\mathfrak{N} \subseteq \mathfrak{A}, \mathfrak{B} \subseteq \mathfrak{M}$ are independent over $\mathfrak{N}$ if $\mathcal{E}_{\mathfrak{N}}(x y)=\mathcal{E}_{\mathfrak{N}}(x) \mathcal{E}_{\mathfrak{N}}(y)$ for all $x \in \mathfrak{A}, y \in \mathfrak{B}$. In particular, two random variables $X$ and $Y$ of a commutative von Neumann algebra $L^{\infty}(\mu)$ 
in which $\mu$ is a probability measure are independent if the algebras they generate are independent over the complex field $\mathbb{C}$. A sequence of subalgebras $\mathfrak{N} \subseteq \mathfrak{A}_{1}, \ldots, \mathfrak{A}_{n} \subseteq \mathfrak{M}$ is called successively independent over $\mathfrak{N}$ if $\mathfrak{A}_{k+1}$ is independent of the algebra $\mathfrak{M}(k)$ generated by $\mathfrak{A}_{1}, \ldots, \mathfrak{A}_{k}$. For further information the reader is referred to $[\mathbf{1 8}, \mathbf{1 9}]$.

In 1970, Rosenthal [14] presented an inequality to describe isomorphic types of some subspaces in $L_{p}$-spaces. It indeed gives a bound for the $p$-norm of independent mean 0 random variables. More precisely, it says that, for any $p \geq 2$, there exists a constant $c(p)$ such that, for any $n \in \mathbb{N}$ and any independent mean 0 random variables $f_{1}, \cdots, f_{n}$, it holds that

$$
\mathbb{E}\left|\sum_{k=1}^{n} f_{k}\right|^{p} \leq c(p)\left(\left(\sum_{k=1}^{n} \mathbb{E}\left|f_{k}\right|^{2}\right)^{p / 2}+\sum_{k=1}^{n} \mathbb{E}\left|f_{k}\right|^{p}\right) .
$$

Burkholder [3] generalized Rosenthal's inequality in the context of martingales. Since then, this inequality has been generalized and applied by many mathematicians; see, e.g., $[\mathbf{8}, 9]$ and references therein. Recently, Junge and Zeng [10] extended the Bennett and Bernstein inequalities to the noncommutative setting and derive a version of the Rosenthal inequality from Bernstein's inequality by using the properties of Gamma function. In probability theory, the Bennett inequality (Bernstein inequality, respectively) gives an upper bound on the probability that the sum of independent random variables deviates from its expected value (deviates from its mean, respectively) by more than a fixed amount, see [1].

In this paper, we establish an extension of the noncommutative Bennett inequality due to Junge and Zeng [10] and use it together with some noncommutative techniques to prove the Rosenthal inequality with a parameter $1 \leq r \leq 2$. We also prove a noncommutative Hoeffding inequality. The Hoeffding inequality [6] gives a probability bound for the deviation between the average of $n$ independent bounded random variables and its mean (see Corollary 3.4). There have been several generalizations and applications of this significant inequality, see $[2,17]$.

2. Bennet inequality. We provide an improved version of the noncommutative Bennett inequality based on the arguments of $[\mathbf{1 0}$, Theorem 01]. 
Theorem 2.1 (Noncommutative Bennett inequality). Let $\mathfrak{N} \subseteq \mathfrak{A}_{j} \subseteq$ $\mathfrak{M}$ be successively independent over $\mathfrak{N}$, and let $x_{j} \in \mathfrak{A}_{j}$ be self-adjoint and $1 \leq r \leq 2$ such that

- $\mathcal{E}_{\mathfrak{N}}\left(x_{j}\right) \leq 0$,

- $\mathcal{E}_{\mathfrak{N}}\left(\left|x_{j}\right|^{r}\right) \leq b_{j}^{r}$,

- $\left\|x_{j}\right\| \leq M$,

for some $M>0$ and all $1 \leq j \leq n$. Then, for each $t \geq 0$,

$$
\operatorname{Prob}\left(\sum_{j=1}^{n} x_{j} \geq t\right)=\tau\left(\chi_{[t, \infty)}\left(\sum_{j=1}^{n} x_{j}\right)\right) \leq \exp \left(\frac{-b}{M^{r}} \Phi\left(\frac{t M^{r-1}}{b}\right)\right),
$$

where $\Phi(\alpha)=(1+\alpha) \log (1+\alpha)-\alpha$ and $b=\sum_{j=1}^{n} b_{j}^{r}$.

Proof. Let $\lambda \geq 0$. We have

$$
\begin{aligned}
\mathcal{E}_{\mathfrak{N}}\left(e^{\lambda x_{n}}\right) & =\mathcal{E}_{\mathfrak{N}}\left(\sum_{k=0}^{\infty} \frac{\left(\lambda x_{n}\right)^{k}}{k !}\right)=\sum_{k=0}^{\infty} \frac{\lambda^{k}}{k !} \mathcal{E}_{\mathfrak{N}}\left(x_{n}^{k}\right) \\
& =1+\mathcal{E}_{\mathfrak{N}}\left(x_{n}\right)+\sum_{k=2}^{\infty} \frac{\lambda^{k}}{k !} \mathcal{E}_{\mathfrak{N}}\left(x_{n}^{k}\right) \\
& \leq 1+\sum_{k=2}^{\infty} \frac{\lambda^{k}}{k !} \mathcal{E}_{\mathfrak{N}}\left(x_{n}^{k}\right) \quad\left(\text { by } \mathcal{E}_{\mathfrak{N}}\left(x_{n}\right) \leq 0\right) \\
& \leq 1+\sum_{k=2}^{\infty} \frac{\lambda^{k}}{k !} \mathcal{E}_{\mathfrak{N}}\left(\left|x_{n}\right|^{k}\right)\left(\mathrm{by} x_{n}^{k} \leq\left|x_{n}\right|^{k}\right) \\
& =1+\sum_{k=2}^{\infty} \frac{\lambda^{k}}{k !} \mathcal{E}_{\mathfrak{N}}\left(\left|x_{n}\right|^{r}\left|x_{n}\right|^{k-r}\right) \\
& \leq 1+\sum_{k=2}^{\infty} \frac{\lambda^{k}}{k !}\left\|x_{n}\right\|^{k-r} \mathcal{E}_{\mathfrak{N}}\left(\left|x_{n}\right|^{r}\right) \\
& \leq 1+\frac{b_{n}^{r}}{\left.\left\|x_{n}\right\|\right|^{r}}\left(e^{\lambda\left\|x_{n}\right\|}-1-\lambda\left\|x_{n}\right\|\right) \\
& \leq 1+\sum_{k=2}^{\infty} \frac{\lambda^{k}}{k !}\left\|x_{n}\right\|^{k-r} b_{n}^{r} \\
& \left.=\left.1 x_{n}\right|^{r}\left|x_{n}\right|^{k-r} \leq\left\|x_{n}\right\|^{k-r}\left|x_{n}\right|^{r}\right) \\
&
\end{aligned}
$$




$$
\leq \exp \left(\frac{b_{n}^{r}}{\left\|x_{n}\right\|^{r}}\left(e^{\lambda\left\|x_{n}\right\|}-1-\lambda\left\|x_{n}\right\|\right)\right) .
$$

Note that the function $f(s):=\exp \left(e^{\lambda s}-1-\lambda s / s^{r}\right)$ is increasing for $s>0$. It follows that

$$
\mathcal{E}_{\mathfrak{N}}\left(e^{\lambda x_{n}}\right) \leq \exp \left(\frac{b_{n}^{r}}{M^{r}}\left(e^{\lambda M}-1-\lambda M\right)\right) .
$$

It follows from (1.2) that

$$
\begin{aligned}
\tau\left(\chi_{[t, \infty)}\left(\sum_{j=1}^{n} x_{j}\right)\right) & =\tau\left(\chi_{[\lambda t, \infty)}\left(\sum_{j=1}^{n} \lambda x_{j}\right)\right) \\
& \leq \exp (-\lambda t) \tau\left(e^{\sum_{j=1}^{n} \lambda x_{j}}\right) .
\end{aligned}
$$

Recall that the Golden-Thompson inequality [15] states that, for all self-adjoint elements $z_{1}, z_{2} \in \mathfrak{M}$,

$$
\tau\left(e^{z_{1}+z_{2}}\right) \leq \tau\left(e^{z_{1}} e^{z_{2}}\right) .
$$

Hence,

$$
\begin{aligned}
\tau\left(e^{\sum_{j=1}^{n} \lambda x_{j}}\right) & \leq \tau\left(e^{\sum_{j=1}^{n-1} \lambda x_{j}} e^{\lambda x_{n}}\right) \quad(\text { by }(2.4)) \\
& =\tau\left(\mathcal{E}_{\mathfrak{N}}\left(e^{\sum_{j=1}^{n-1} \lambda x_{j}} e^{\lambda x_{n}}\right)\right) \\
& =\tau\left(\mathcal{E}_{\mathfrak{N}}\left(e^{\sum_{j=1}^{n-1} \lambda x_{j}}\right) \mathcal{E}_{\mathfrak{N}}\left(e^{\lambda x_{n}}\right)\right) \\
& \leq \exp \left(\frac{b_{n}^{r}}{M^{r}}\left(e^{\lambda M}-1-\lambda M\right)\right) \tau\left(\mathcal{E}_{\mathfrak{N}}\left(e^{\sum_{j=1}^{n-1} \lambda x_{j}}\right)\right) \\
& \leq \exp \left(\frac{\sum_{j=1}^{n} b_{j}^{r}}{M^{r}}\left(e^{\lambda M}-1-\lambda M\right)\right)
\end{aligned}
$$

(by iterating $n-2$ times)

We infer from the latter inequality together with (2.3) that

$$
\tau\left(\chi_{[t, \infty)}\left(\sum_{j=1}^{n} x_{j}\right)\right) \leq \exp \left(-\lambda t+\frac{\sum_{j=1}^{n} b_{j}^{r}}{M^{r}}\left(e^{\lambda M}-1-\lambda M\right)\right) .
$$


By the basic calculus method we find the minimizing value

$$
\lambda=\frac{1}{M} \log \left(1+\frac{t M^{r-1}}{\sum_{j=1}^{n} b_{j}^{r}}\right),
$$

which yields (2.1).

Since both inequalities $\Phi(t) \geq t^{2} / 2+2 t / 3$ and $\Phi(t) \geq t / 2 \operatorname{arcsinh}(t / 2)$ are valid for all $t \geq 0$, one can get the Bernstein and Prohorov inequalities from Bennett's inequality as follows.

Corollary 2.2. Under the same hypothesis as Theorem 2.1,

$$
\tau\left(\chi_{[t, \infty)}\left(\sum_{j=1}^{n} x_{j}\right)\right) \leq \exp \left(-\frac{t^{2} M^{r-2}}{2 b+2 / 3 t M^{r-1}}\right)
$$

and

$$
\tau\left(\chi_{[t, \infty)}\left(\sum_{j=1}^{n} x_{j}\right)\right) \leq \exp \left(-\frac{t}{2 M} \operatorname{arcsinh}\left(\frac{t M}{2 b}\right)\right)
$$

We can immediately deduce the following commutative Bernstein's inequality.

Corollary 2.3. Let $X_{1}, \ldots, X_{n}$ be independent Bernoulli random variables taking values 1 and -1 with probability $1 / 2$. Then

$$
\operatorname{Prob}\left(\left|\frac{1}{n} \sum_{i=1}^{n} X_{i}\right| \geq \varepsilon\right) \leq 2 e^{-n \varepsilon^{2} / 2+(2 \varepsilon / 3)} .
$$

3. Hoeffding inequality. In this section, we provide a noncommutative version of Hoeffding's inequality and present some consequences.

Theorem 3.1 (Noncommutative Hoeffding inequality). Let $\mathfrak{N} \subseteq \mathfrak{A}_{j} \subseteq$ $\mathfrak{M}$ be successively independent over $\mathfrak{N}$, and let $x_{j} \in \mathfrak{A}_{j}$ be self-adjoint such that $a_{j} \leq x_{j} \leq b_{j}$ for some real numbers $a_{j}<b_{j}$ and $\mathcal{E}_{\mathfrak{N}}\left(x_{j}\right)=\mu$ for some $\mu \geq 0$ and all $1 \leq j \leq n$. Then

$$
\operatorname{Prob}\left(\left|S_{n}-n \mu\right| \geq t\right) \leq 2 \exp \left(\frac{-2 t^{2}}{\sum_{j=1}^{n}\left(b_{j}-a_{j}\right)^{2}}\right),
$$


for any $t>0$, where $S_{n}=\sum_{j=1}^{n} x_{j}$.

Proof. First we show that, if $x \in \mathfrak{A}_{j}$ is self-adjoint such that $a \leq x \leq b$ and $\mathcal{E}_{\mathfrak{N}}(x)=0$, then

$$
\mathcal{E}_{\mathfrak{N}}\left(e^{s x}\right) \leq \exp \left(\frac{s^{2}(b-a)^{2}}{8}\right)
$$

for any $s>0$.

Let $s>0$. Note that $t \mapsto e^{t s}$ is convex; therefore, for any $a \leq \alpha \leq b$,

$$
e^{s \alpha} \leq e^{s b} \frac{\alpha-a}{b-a}+e^{s a} \frac{b-\alpha}{b-a} .
$$

By functional calculus, we have

$$
e^{s x} \leq e^{s b} \frac{x-a}{b-a}+e^{s a} \frac{b-x}{b-a} .
$$

Since $\mathcal{E}_{\mathfrak{N}}$ is a positive map and $\mathcal{E}_{\mathfrak{N}}(x)=0$, we reach

$$
\mathcal{E}_{\mathfrak{N}}\left(e^{s x}\right) \leq \frac{-a}{b-a} e^{s b}+\frac{b}{b-a} e^{s a}=e^{h(\alpha)},
$$

where $\alpha=s(b-a), h(\alpha)=-\gamma \alpha+\log \left(1-\gamma+\gamma e^{\alpha}\right)$ and $\gamma=-a /(b-a)$. In addition, $h(0)=h^{\prime}(0)=0$ and $h^{\prime \prime}(\alpha) \leq 1 / 4$ for all $\alpha>0$. By Taylor's theorem, there exists a real number $\xi \in(0, \alpha)$ such that

$$
h(\alpha)=h(0)+\alpha h^{\prime}(0)+\frac{\alpha^{2}}{2} h^{\prime \prime}(\xi) \leq \frac{\alpha^{2}}{8}=\frac{s^{2}(b-a)^{2}}{8} .
$$

Hence,

$$
\mathcal{E}_{\mathfrak{N}}\left(e^{s x}\right) \leq \exp \left(\frac{s^{2}(b-a)^{2}}{8}\right) .
$$

Second, for arbitrary values of $\mathcal{E}_{\mathfrak{N}}(x)$, setting $y:=x-\mu$, we get $a-\mu \leq y \leq b-\mu$ and $\mathcal{E}_{\mathfrak{N}}(y)=0$. Employing (3.2), we reach

$$
\mathcal{E}_{\mathfrak{N}}\left(e^{s(x-\mu)}\right) \leq \exp \left(\frac{s^{2}(b-a)^{2}}{8}\right) \text {. }
$$

Next, by the same reasoning as in the proof of Theorem 2.1, we get from (3.3) that

$$
\tau\left(e^{\lambda \sum_{j=1}^{n}\left(x_{j}-\mu\right)}\right) \leq \tau\left(\mathcal{E}_{\mathfrak{N}}\left(e^{\lambda \sum_{j=1}^{n-1}\left(x_{j}-\mu\right)}\right) \mathcal{E}_{\mathfrak{N}}\left(e^{\lambda\left(x_{n}-\mu\right)}\right)\right)
$$




$$
\begin{aligned}
& \leq e^{\lambda^{2}\left(b_{n}-a_{n}\right)^{2} / 8} \tau\left(\mathcal{E}_{\mathfrak{N}}\left(e^{\lambda \sum_{j=1}^{n-1}\left(x_{j}-\mu\right)}\right)\right) \leq \cdots \\
& \leq \exp \left(\frac{\lambda^{2} \sum_{j=1}^{n}\left(b_{j}-a_{j}\right)^{2}}{8}\right)
\end{aligned}
$$

Therefore, (1.1) and the exponential Chebyshev inequality (1.2) yield that

$$
\begin{aligned}
\operatorname{Prob}\left(\left|S_{n}-n \mu\right| \geq t\right) & =2 \operatorname{Prob}\left(S_{n}-n \mu \geq t\right) \\
& \leq 2 e^{-\lambda t} \exp \left(\frac{\lambda^{2} \sum_{j=1}^{n}\left(b_{j}-a_{j}\right)^{2}}{8}\right) .
\end{aligned}
$$

This is minimized when $\lambda=4 t / \sum_{j=1}^{n}\left(b_{j}-a_{j}\right)^{2}$. Thus,

$$
\operatorname{Prob}\left(\left|S_{n}-n \mu\right| \leq 2 \exp \left(\frac{-2 t^{2}}{\sum_{j=1}^{n}\left(b_{j}-a_{j}\right)^{2}}\right)\right.
$$

which is the desired inequality.

Remark 3.2. Under the same hypothesis as Theorem 3.1 we can show that the bound in the Hoeffding inequality (3.1) is sharper than that in the Bernstein inequality (2.5) for $r=2$. Let us assume that $\mathcal{E}_{\mathfrak{N}}\left(x_{j}\right)=0$ and $-1 \leq x_{j} \leq 1$. By the functional calculus $\left|x_{j}\right| \leq 1$ so $\mathcal{E}_{\mathfrak{N}}\left(\left|x_{j}\right|^{2}\right) \leq 1$ and $\left\|x_{j}\right\| \leq 1$, therefore $b=n$ and $M=1$ in the notation of Theorem 2.1. Then the Heoffding inequality gives rise to

$$
\operatorname{Prob}\left(\sum_{j=1}^{n} x_{j} \geq t\right) \leq \exp \left(\frac{-t^{2}}{2 n}\right)
$$

and, from the Bernstein inequality, we have

$$
\operatorname{Prob}\left(\sum_{j=1}^{n} x_{j} \geq t\right) \leq \exp \left(\frac{-t^{2}}{2 n+(2 t / 3)}\right) .
$$

The next result is the classical (commutative) version of the Hoeffding inequality.

Corollary 3.3 (Hoeffding's inequality [6]). Let $a \leq X_{1}, \ldots, X_{n} \leq b$ be independent random variables with the expectation $\mathbb{E}\left(X_{i}\right)=\mu$, 


$$
\begin{aligned}
& i=1, \ldots, n . \text { If } \bar{X}_{n}=\left(\sum_{i=1}^{n} X_{i}\right) / n \text {, then } \\
& \operatorname{Prob}\left(\left|\bar{X}_{n}-\mu\right| \geq t\right) \leq 2 e^{-2 n t^{2} /(b-a)^{2}} .
\end{aligned}
$$

In the special case, we immediately get

Corollary 3.4. Let $0 \leq X_{1}, \ldots, X_{n} \leq 1$ be independent random variables with common mean $\mu$. Then with probability at least $1-\varepsilon$,

$$
\left|\frac{1}{n} \sum_{i=1}^{n} X_{i}-\mu\right| \leq \sqrt{\frac{\log (2 / \varepsilon)}{2 n}} .
$$

4. Rosenthal inequality. In this section, we intend to prove a noncommutative Rosenthal inequality by using our noncommutative Bennet inequality. Our argument seems to be simpler than that of [7] for the case of usual random variables. We note that there is a refinement of it in the literature in which various approaches are used, see [10, Theorem 0.4] and [5].

Theorem 4.1. Let $1 \leq r \leq 2 \leq p<\infty, \mathfrak{N} \subseteq \mathfrak{A}_{j} \subseteq \mathfrak{M}$ be successively independent over $\mathfrak{N}$, and let $x_{j} \in \mathfrak{A}_{j}$ be self-adjoint such that $\mathcal{E}_{\mathfrak{N}}\left(x_{j}\right)=0$. Then there exists a constant $C(p, r)$ such that

$$
\left\|\sum_{j=1}^{n} x_{j}\right\|_{p}^{p} \leq C(p, r)\left\{\sum_{j=1}^{n}\left\|x_{j}\right\|_{p}^{p}+\left(\sum_{j=1}^{n}\left\|x_{j}^{r}\right\|\right)^{p / r}\right\} .
$$

Proof. We use the noncommutative Bennett inequality, but, for this end, we replace $\Phi(\alpha)$ by $\alpha \log (1+\alpha)-\alpha$, which is clearly smaller than $\Phi(\alpha)$ for any $\alpha \geq 0$.

Let us fix an arbitrary number $s \geq 0$, and consider $y_{j}=x_{j} \chi_{(-\infty, s]}\left(x_{j}\right)$ $\in \mathfrak{A}_{j}$. It follows from $y_{j} \leq x_{j}$ and the positivity of $\mathcal{E}_{\mathfrak{N}}$ that $\mathcal{E}_{\mathfrak{N}}\left(y_{j}\right) \leq$ $\mathcal{E}_{\mathfrak{N}}\left(x_{j}\right)=0$. In addition, $\mathcal{E}_{\mathfrak{N}}$ is norm decreasing, so

$$
\sum_{j=1}^{n} \mathcal{E}_{\mathfrak{N}}\left(\left|x_{j}\right|^{r}\right) \leq \sum_{j=1}^{n}\left\|x_{j}^{r}\right\|:=B
$$


and

$$
b:=\sum_{j=1}^{n}\left\|y_{j}^{r}\right\| \geq \sum_{j=1}^{n} \mathcal{E}_{\mathfrak{N}}\left(\left|y_{j}\right|^{r}\right) .
$$

Further, $b \leq B$ since $\left\|y_{j}\right\| \leq\left\|x_{j}\right\| \leq M$, where $M:=\max _{1 \leq j \leq n}\left\|x_{j}\right\|$. It follows from the noncommutative Bennett inequality (2.1) that

$$
\begin{aligned}
\tau\left(\chi_{[t, \infty)} \sum_{j=1}^{n} y_{j}\right) & \leq \exp \left(\frac{-b}{M^{r}} \Phi\left(\frac{t M^{r-1}}{b}\right)\right) \\
& \leq \exp \left(\frac{-b}{M^{r}}\left[\frac{t M^{r-1}}{b} \log \left(1+\frac{t M^{r-1}}{b}\right)-\frac{t M^{r-1}}{b}\right]\right) \\
& \leq \exp \left(-\frac{t}{M}\left(\log \left(1+\frac{t M^{r-1}}{B}\right)-1\right)\right)
\end{aligned}
$$

for all $t>0$. We have

$$
\chi_{[t, \infty)}\left(\sum_{j=1}^{n} x_{j}\right) \prec \chi_{[t, \infty)}\left(\sum_{j=1}^{n} y_{j}\right) \vee\left(\vee_{j=1}^{n} \chi_{[s, \infty)}\left(x_{j}\right)\right)
$$

for all $t>0$. To show this, we have to prove that

$$
\chi_{[t, \infty)}\left(\sum_{j=1}^{n} x_{j}\right) \wedge\left(\chi_{[0, t)}\left(\sum_{j=1}^{n} y_{j}\right) \wedge\left(\wedge_{j=1}^{n} \chi_{(-\infty, s)}\left(x_{j}\right)\right)\right)=0 .
$$

Let

$$
\begin{array}{r}
\xi \in \chi_{[t, \infty)}\left(\sum_{j=1}^{n} x_{j}\right)(\mathfrak{H}) \bigcap\left(\chi_{[0, t)}\left(\sum_{j=1}^{n} y_{j}\right)\right. \\
\left.(\mathfrak{H}) \bigcap\left(\cap_{j=1}^{n} \chi_{(-\infty, s)}\left(x_{j}\right)(\mathfrak{H})\right)\right) .
\end{array}
$$

Then

$$
\left\langle\left(\sum_{j=1}^{n} x_{j}\right) \xi, \xi\right\rangle \geq t
$$

and

$$
t>\left\langle\left(\sum_{j=1}^{n} y_{j}\right) \xi, \xi\right\rangle=\left\langle\left(\sum_{j=1}^{n} x_{j} \chi_{(-\infty, s)}\left(x_{j}\right)\right) \xi, \xi\right\rangle
$$




$$
=\left\langle\left(\sum_{j=1}^{n} x_{j}\right) \xi, \xi\right\rangle \geq t
$$

since $\xi \in \cap_{j=1}^{n} \chi_{(-\infty, s)}\left(x_{j}\right)(\mathfrak{H})$. Therefore,

$$
\chi_{[t, \infty)}\left(\sum_{j=1}^{n} x_{j}\right) \wedge\left(\chi_{[0, t)}\left(\sum_{j=1}^{n} y_{j}\right) \wedge\left(\wedge_{j=1}^{n} \chi_{(-\infty, s)}\left(x_{j}\right)\right)\right)=0 .
$$

We deduce from Lemma 1.1 (ii) that

$$
\chi_{[t, \infty)}\left(\sum_{j=1}^{n} x_{j}\right) \prec\left(\chi_{[0, t)}\left(\sum_{j=1}^{n} y_{j}\right) \wedge\left(\wedge_{j=1}^{n} \chi_{(-\infty, s)}\left(x_{j}\right)\right)\right)^{\perp},
$$

and this gives us inequality (4.2). Using (4.2), we get

$$
\begin{aligned}
\tau\left(\chi_{[t, \infty)}\left(\sum_{j=1}^{n} x_{j}\right)\right) \leq & \tau\left(\chi_{[t, \infty)}\left(\sum_{j=1}^{n} y_{j}\right)\right)+\tau\left(\vee_{j=1}^{n} \chi_{[s, \infty)}\left(x_{j}\right)\right) \\
& (\text { by Lemma 1.1 (ii)-(iii)) } \\
\leq & \exp \left(-\frac{t}{M}\left(\log \left(1+\frac{t M^{r-1}}{B}\right)-1\right)\right) \\
& +\sum_{j=1}^{n} \tau\left(\chi_{[s, \infty)}\left(x_{j}\right)\right)
\end{aligned}
$$

(by Lemma 1.1 (iv), (4.1))

for any $t>0$. An easy investigation shows that the latter inequality holds if we replace $M$ by any number $L$ with $L \geq M$. In addition, it holds for all $0<L \leq M$ since the function

$$
f(\alpha)=\exp \left(-\frac{t}{\alpha}\left(\log \left(1+\frac{t \alpha^{r-1}}{B}\right)-1\right)\right)
$$

is decreasing for any $\alpha>0$. Thus

$$
\begin{aligned}
\tau\left(\chi_{[t, \infty)}\left(\sum_{j=1}^{n} x_{j}\right)\right) \leq & \exp \left(-\frac{t}{L}\left(\log \left(1+\frac{t L^{r-1}}{B}\right)-1\right)\right) \\
& +\sum_{j=1}^{n} \tau\left(\chi_{[s, \infty)}\left(x_{j}\right)\right)
\end{aligned}
$$

for all $t>0, s>0, L>0$. 
Next, we deal with the modulus of $\sum_{j=1}^{n} x_{j}$. Inequality (4.3) together with (1.1) imply that

$$
\begin{aligned}
\tau\left(\chi_{[t, \infty)}\left(\left|\sum_{j=1}^{n} x_{j}\right|\right)\right) \leq & 2 \exp \left(-\frac{t}{L}\left(\log \left(1+\frac{t L^{r-1}}{B}\right)-1\right)\right) \\
& +\sum_{j=1}^{n} \tau\left(\chi_{[s, \infty)}\left(\left|x_{j}\right|\right)\right) .
\end{aligned}
$$

Now, by putting first $L=s$ and $s=t / \gamma$, where $\gamma>0$ we obtain that

$$
\begin{aligned}
\tau\left(\chi_{[t, \infty)}\left(\left|\sum_{j=1}^{n} x_{j}\right|\right)\right) \leq & 2 \exp \left(-\gamma\left(\log \left(1+\frac{t^{r}}{\gamma^{r-1} B}\right)-1\right)\right) \\
& +\sum_{j=1}^{n} \tau\left(\chi_{[t, \infty)}\left(\gamma\left|x_{j}\right|\right)\right) .
\end{aligned}
$$

For $p \geq 2$, we have

$$
\begin{aligned}
\left\|\sum_{j=1}^{n} x_{j}\right\|_{p}^{p}= & \int_{0}^{\infty} p t^{p-1} \tau\left(\chi_{[t, \infty)}\left(\left|\sum_{j=1}^{n} x_{j}\right|\right)\right) d t \\
\leq & \sum_{j=1}^{n} \int_{0}^{\infty} p t^{p-1} \tau\left(\chi_{[t, \infty)}\left(\gamma\left|x_{j}\right|\right)\right) d t \\
& +2 p \int_{0}^{\infty} t^{p-1} \exp \left(-\gamma\left(\log \left(1+\frac{t^{r}}{\gamma^{r-1} B}\right)-1\right)\right) d t \\
\leq & \gamma^{p} \sum_{j=1}^{n}\left\|x_{j}\right\|_{p}^{p}+\frac{2 p}{r}\left(\gamma^{r-1} B\right)^{p / r} e^{\gamma} \int_{0}^{\infty} \beta^{(p-r) / r}(1+\beta)^{-\gamma} d \beta,
\end{aligned}
$$

where we use the change of variables $\beta=t^{r} / \gamma^{r-1} B$. Next let $\gamma$ be such that the last integral of the above inequality is convergent, i.e., let us choose $\gamma>p / r$. With this choice of $\gamma$, inequality (4.4) implies the Rosenthal inequality with

$$
C(p, r)=\max \left\{\gamma^{p}, \frac{2 p}{r} \gamma^{\frac{p(r-1)}{r}} e^{\gamma} \int_{0}^{\infty} \beta^{p-r / r}(1+\beta)^{-\gamma} d \beta\right\} .
$$

In the commutative setting, we have 
Corollary 4.2. Let $1 \leq r \leq 2 \leq p<\infty$ and $X_{1}, \ldots, X_{n}$ be independent real random variables with expected values $\mathbb{E}\left(X_{j}\right)=0, j=1, \ldots, n$. Then

$$
\mathbb{E}\left(\left|\sum_{j=1}^{n} X_{j}\right|^{p}\right) \leq C(p)\left\{\sum_{j=1}^{n} \mathbb{E}\left(\left|X_{j}\right|^{p}\right)+\left(\sum_{j=1}^{n} \mathbb{E}\left(\left|X_{j}\right|^{r}\right)\right)^{p / r}\right\},
$$

where

$$
C(p)=\min _{\gamma>p / 2} \max \left\{\gamma^{p}, \frac{2 p}{r} \gamma^{p(r-1) / r} e^{\gamma} \int_{0}^{\infty} \beta^{p-r / r}(1+\beta)^{-\gamma} d \beta\right\} .
$$

Acknowledgments. The first author thanks the Tusi Mathematical Research Group (TMRG), Mashhad, Iran. We thank S. Dirksen for pointing out the reference [6] to our attention.

\section{REFERENCES}

1. G. Bennett, Probability inequalities for the sum of independent random variables, J. Amer. Stat. Assoc. 57 (1962), 33-45.

2. V. Bentkus, On Hoeffding's inequalities, Ann. Prob. 32 (2004), 1650-1673.

3. D.L. Burkholder, Distribution function inequalities for martingales, Ann. Prob. 1 (1973), 19-42.

4. J.B. Conway, A course in functional analysis, Grad. Texts Math., SpringerVerlag, New York, 1990.

5. S. Dirksen, B. de Pagter, D. Potapov and F. Sukochev, Rosenthal inequalities in noncommutative symmetric spaces, J. Funct. Anal. 261 (2011), 2890-2925.

6. W. Hoeffding, Probability inequalities for sums of bounded random variables, J. Amer. Stat. Assoc. 58 (1963), 13-30.

7. X.-L. Hu, An extension of Rosenthal's inequality, Appl. Math. Comp. 218 (2011), 4638-4640.

8. W.B. Johnson, G. Schechtman and J. Zinn, Best constants in moment inequalities for linear combinations of independent and exchangeable random variables, Ann. Prob. 13 (1985), 234-253.

9. M. Junge and Q. Xu, Noncommutative Burkholder/Rosenthal inequalities, II, Applications, Israel J. Math. 167 (2008), 227-282.

10. M. Junge and Q. Zeng, Noncommutative Bennett and Rosenthal inequalities, Ann. Prob. 41 (2013), 4287-4316.

11. M.S. Moslehian, M. Tominaga and K.-S. Saito, Schatten p-norm inequalities related to an extended operator parallelogram law, Linear Alg. Appl. 435 (2011), 823-829.

12. E. Nelson, Note on noncommutative integration, J. Funct. Anal. 15 (1974), $103-116$. 
13. B. de Pagter, Noncommutative Banach function spaces, Positivity, 197-227, Birkhüser, Basel, 2007.

14. H.P. Rosenthal, On the subspaces of $L_{p}(p>2)$ spanned by sequences of independent random variables, Israel J. Math. 8 (1970), 273-303.

15. M.B. Ruskai, Inequalities for traces on von Neumann algebras, Comm. Math. Phys. 26 (1972), 280-289.

16. Gh. Sadeghi, Non-commutative Orlicz spaces associated to a modular on $\tau$-measurable operators, J. Math. Anal. Appl. 395 (2012), 705-715.

17. J.A. Tropp, User-friendly tail bounds for sums of random matrices, Found. Comp. Math. 12 (2012), 389-434.

18. Q. Xu, Embedding of $C_{q}$ and $R_{q}$ into noncommutative $L_{p}$-spaces, $1 \leq p<$ $q \leq 2$, Math. Ann. 335 (2006), 109-131.

19. Operator spaces and noncommutative $L_{p}$, Lect. Summer School Banach Spaces Operator Spaces, Nankai University, China, 2007.

20. F.J. Yeadon, Noncommutative $L^{p}$-spaces, Proc. Camb. Phil. Soc. 77 (1975), 91-102.

Department of Mathematics and Computer Sciences, Hakim Sabzevari UniVERSity, P.O. Box 397, SABZEVAR, Iran

Email address: ghadir54@gmail.com, g.sadeghi@hsu.ac.ir

Department of Pure Mathematics, Center of Excellence in Analysis on Algebraic Structures (CEAas), Ferdowsi University of Mashhad, P.O. Box 1159, MashHad 91775, Iran

Email address: moslehian@um.ac.ir, moslehian@member.ams.org 